\title{
Numerical and experimental analysis of the velocity field of air flowing through swirl diffusers
}

\author{
Marek Borowski ${ }^{1, *}$, Michat Karch $^{1}$, Rafał Łuczak $^{1}$, Piotr Życzkowski ${ }^{1}$, and Marek Jaszczur ${ }^{1}$ \\ ${ }^{1}$ AGH University of Science and Technology, 30 Mickiewicza av., 30-059 Kraków, Poland
}

\begin{abstract}
The air swirl diffusers are popular for the ceiling level air supply system and have been widely used for Indoor Air Quality. They are nowadays one of the most popular diffusers commonly used in airconditioning systems $[1,2]$. The swirl diffusers are intended to use in low- and medium-pressure ventilation systems. They allow for obtaining the swirl airflow and are especially recommended to use in spaces with the height from $3 \mathrm{~m}$ up to $12 \mathrm{~m}$, where heating and cooling is performed by the ventilation system and where the exact setting of supply air velocity is important [3-6]. In the case of swirl diffusers, fluid flow is usually significantly influenced by the characteristics of different diffuser designs. In the ventilation system design phase, a typically focus on ventilation effectiveness and takes into account a large number of parameter. However, the discrepancy between design and practice are often experienced mainly because operating conditions don't correspond to the designing conditions [7-9]. In this work, the airflow of swirl diffuser has been studied using Particle Image Velocimetry (PIV) method to analyse the mean airflow and geometry influence on the results. The paper presents a comparison of the results of velocity field measurements using the PIV method and the results obtained by means of numerical analysis CFD. Based on the analysis the velocity flow field was evaluated and the range of effective operation of the diffuser have been determined [10].
\end{abstract}

\section{Introduction}

The efficiency of the ventilation system depends on the proper distribution of air in the room. To ensure the necessary conditions, the required ventilation effects can be determined in various ways. You can specify the spatial temperature distribution and range of air velocity in a certain zone or in the whole room. The distribution of pollutant concentrations can also be determined. You can also specify the number of air changes in the room. Given are also recommendations for a geometric division of the room into zones with defined values of flow and thermal parameters. The expected effects of ventilation should be related to the impact of local factors in a given facility. Determination of the required ventilation effects is based on the indication of a significant and measurable effect of air separation $[1,2]$.

\section{Experimental setup}

For the needs of the research, a model of the installation with a horizontal swirl diffuser was developed (Figure 1). The tested swirl diffuser was made in the 1:10 scale, in three variants differing in the arrangement of the vanes. The vanes were set at 45, 60 and 75 degrees. Analysed diffusers are shown in Figure 2. The air was supplied to the diffuser through the system used to stabilize the stream. It was made of a diffuser, confusor and a $0.2 \mathrm{~m}$ diameter duct. Its length was $1.35 \mathrm{~m}$. The air was then supplied to the diffuser using a $2 \mathrm{~m}$ long channel with a diameter equal to $50 \mathrm{~mm}$. The aim of the research was to experimentally measure the velocity fields of the stream of air blown in by the swirl diffuser. The experimental setup is equipped with the Stereoscopic-DPIV measuring system from Lavision $\mathrm{GmbH}$.

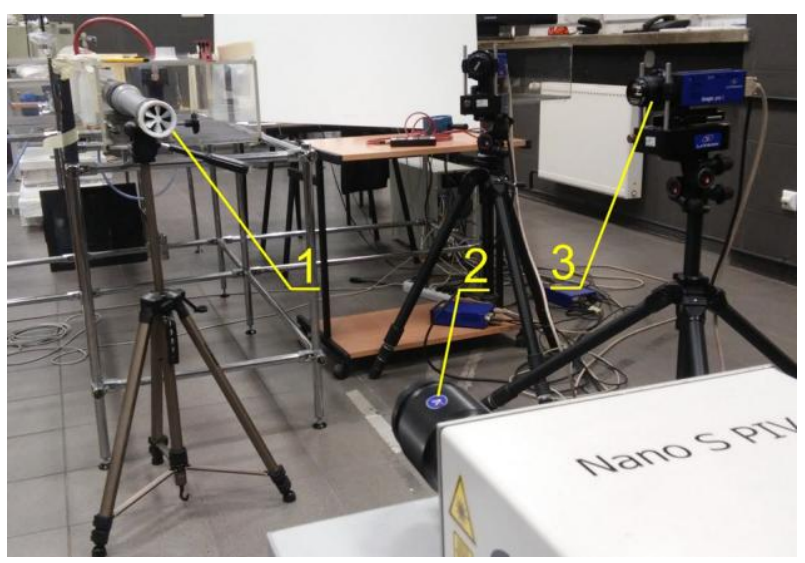

Fig. 1. Set-up of the measurement section: 1- swirl diffuser, 2laser Nd:Yag, 3 - CCD camera.

Digital Particle Image Velocimetry (DPIV) is a noninvasive method for measuring fluid velocity. It allows to determine the distribution of the fluid velocity field in the entire examined measurement area. The size of the

* Corresponding author: borowski@agh.edu.pl 
area is limited only by the field of view of the lens used to record the image and the illumination power used. The DPIV method is presented as a quantitative measurement of fluid velocity in a large number of points [10].

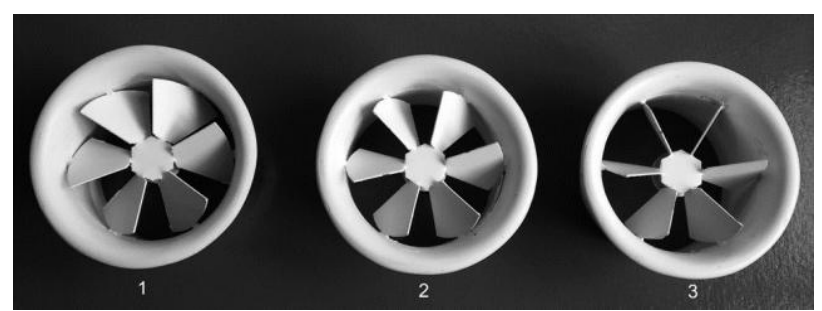

Fig. 2. Diffuser vanes: 1-angle of 45 degrees, 2-angle of 60 degrees and 3 -angle of 75 degrees.

Diethylohexyl-sebacate (DEHS - C26H50O4) was used as a seeding and a 4.0 Megapixels monochrome CCD camera was used. The camera was equipped with a Canon lens with a focal length of $50 \mathrm{~mm}$ and a minimum aperture value of 1.8. The source of light was composed of double-pulse Nd: YAG laser Litron Nano S PIV with a pulse energy of $60 \mathrm{~mJ}$. The laser's operating frequency ranged from $1 \mathrm{~Hz}$ to $15 \mathrm{~Hz}$ and for current purposes frequency $7 \mathrm{~Hz}$ was used. The measuring set-up was described in more detail in $[2,5,6]$. Measurements were made for four values of the average volumetric airflow rate through the diffuser: $100,185,310$ and $380 \mathrm{~m}^{3} / \mathrm{h}$. This corresponds to the values of Reynolds number $\mathrm{Re} \approx 47,000.00, \quad \mathrm{Re} \approx 85,000.00, \quad \operatorname{Re} \approx 143,000.00$ and $\mathrm{Re} \approx 183,000.00$. Two velocity components were determined in the vertical plane passing through the diffuser axis (Figure 3).



Fig. 3. Measurement section with a coordinate system.

\section{Results}

PIV measurement gives very detailed information on velocity component vectors in the entire meaeasurement section. The method allows to determine velocity vector fields as well as the shape of the stream and the main flow directions. In Figures 4-6 velocity vectors and the main airflow stream are visible. The Figures are presented for a different angle of vanes setting and the minimum value of the air stream. It can be seen that the shape of the stream depends on the value of the vanes angle.

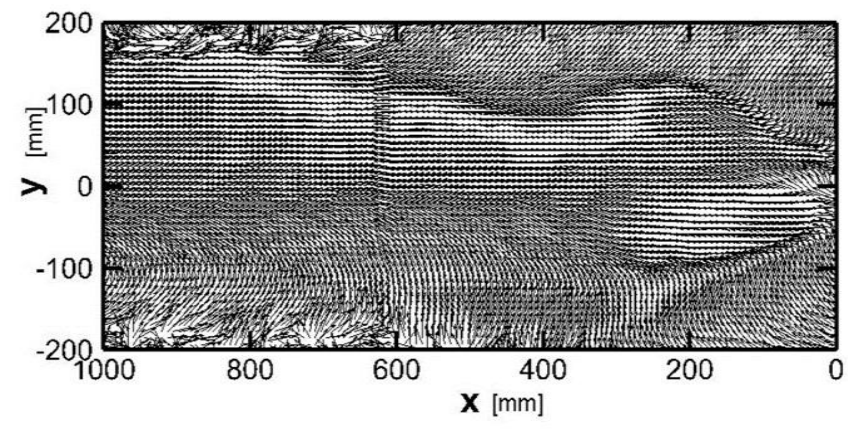

Fig. 4. The measured velocity field. For the case: $45^{\circ}$ diffuser and flowrate $100 \mathrm{~m}^{3} / \mathrm{h}$.

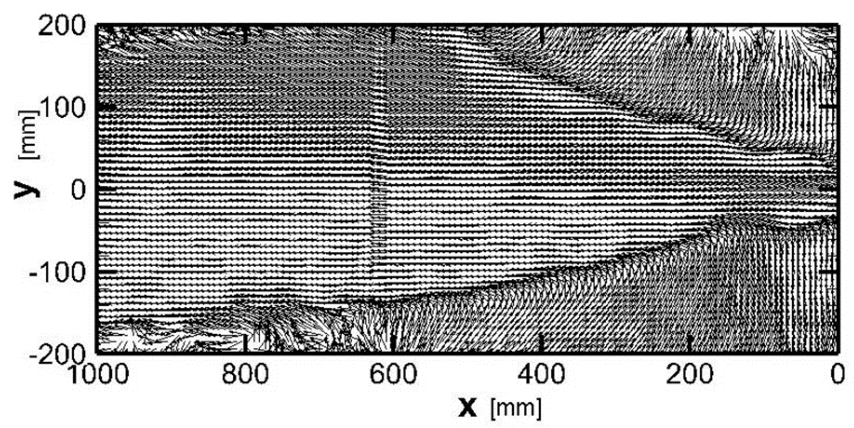

Fig. 5. The measured velocity field. For the case: $60^{\circ}$ diffuser and flowrate $100 \mathrm{~m}^{3} / \mathrm{h}$.

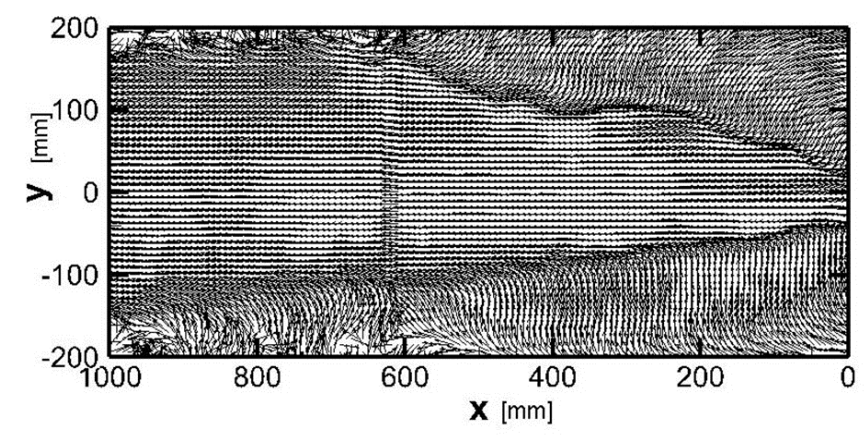

Fig. 6. The measured velocity field. For the case: $75^{\circ}$ diffuser and flowrate $100 \mathrm{~m}^{3} / \mathrm{h}$.

\section{Analysis}

Fig. 7 shows normalized by the inlet velocity flow fields for vanes angle set-up to 60 degrees, obtained for flows with Reynolds numbers: $\operatorname{Re}_{1}=47,000.00, \operatorname{Re}_{2}=85,000.00$, $\mathrm{Re}_{3}=143,000.00, \mathrm{Re}_{4}=183,000.00$. The comparison of these distributions shows that for all Reynolds numbers there is an approximate similarity between normed velocity fields. At the same time, it should be noted that the similarity increases with the value of the angle of the diffuser vanes. It should also be noted that the closer the outlet from the diffuser, the greater the compliance of the normalised fields. It is also worth pointing out some limitations of the measurement method itself related to the low resolution of the edges of the studied area. This may result in distortions and "noises" in some areas. This phenomenon can be seen in the Figures 8 (a)-(f). In order to eliminate this phenomenon, the measurement area has been divided into smaller overlapping areas. Due to this 
procedure, it is possible to measure velocity fields with higher resolution. However, this results in the complexity of the measurement process itself.

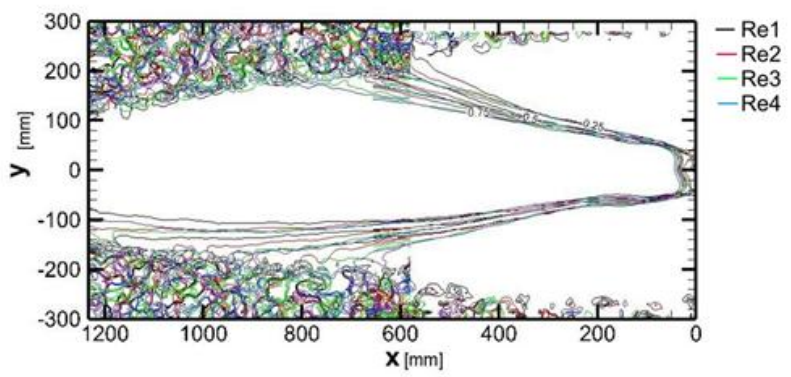

Fig. 7. Diffuser with vanes at an angle of $60^{\circ}$ the image of normalised velocity fields for different Reynolds numbers: $\mathrm{Re}_{1}=47,000.00, \mathrm{Re}_{2}=85,000.00, \mathrm{Re}_{3}=143,000.00$, $\mathrm{Re}_{4}=183,000.00$.

The velocity normalisation was done by dividing the local velocity values by the average velocity value measured at the outlet from the diffuser:

$$
\text { Vnor }=\frac{V}{V_{s r}}
$$

Where:

$V$ - the value of the air velocity in the analyzed measuring section,

$V_{\text {sr }}-$ the value of the average air velocity in the outlet cross-section of the tested diffuser.

The average velocity value was determined based on the volumetric flow volume measurement. This operation aims at comparing the speed profiles obtained at different values of the air stream blown through the diffuser.

Figure 8 shows normalized velocity profiles at different distances from the diffuser outlet with vanes angle equal to $60^{\circ}$. One may infer from this figure a large similarity of profiles obtained for different Reynolds numbers. As the distance from the diffuser increases, the value of the normalised velocity decreases. The velocity profile expands with the distance from the diffuser outlet. Normalised velocity profiles determined closer to the diffuser outlet are more similar than profiles determined at a larger distance.

(a) $\mathrm{x}=70 \mathrm{~mm}$

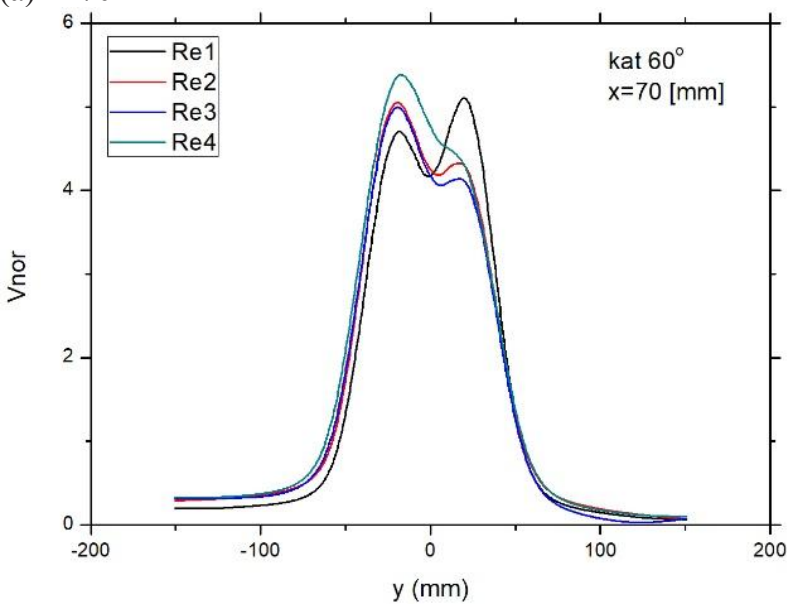

(b) $\mathrm{x}=100 \mathrm{~mm}$

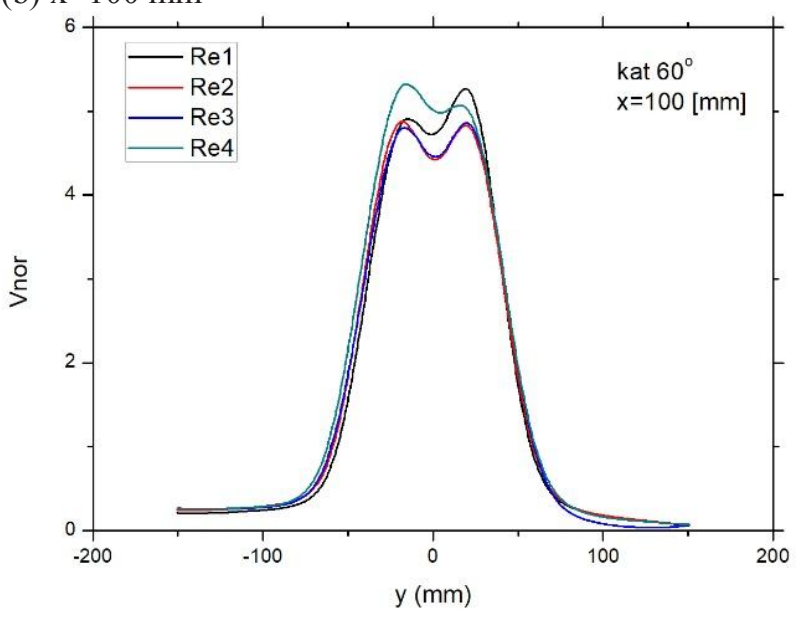

(c) $x=150 \mathrm{~mm}$

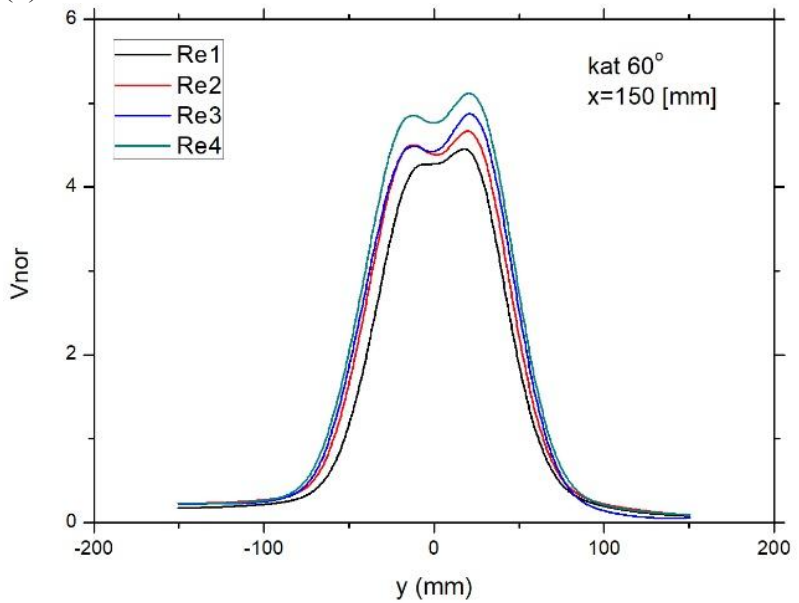

(d) $\mathrm{x}=200 \mathrm{~mm}$

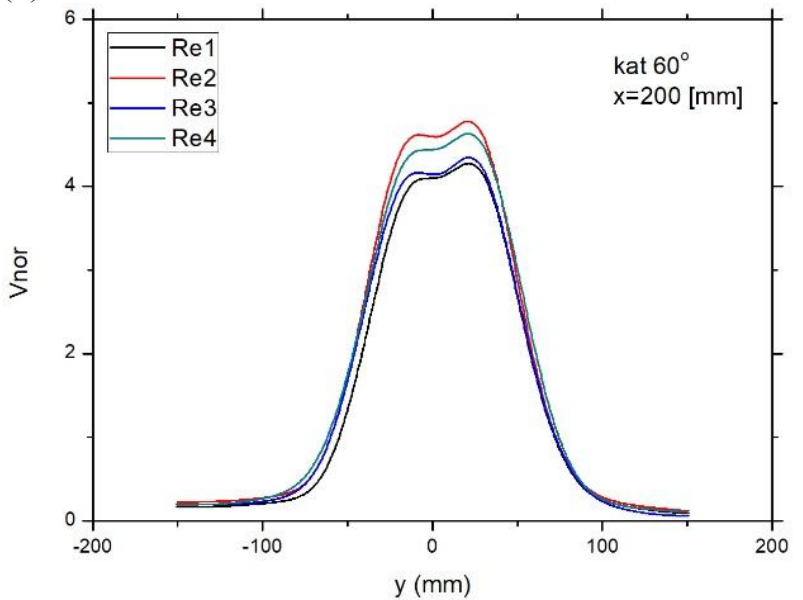


(e) $\mathrm{x}=300 \mathrm{~mm}$

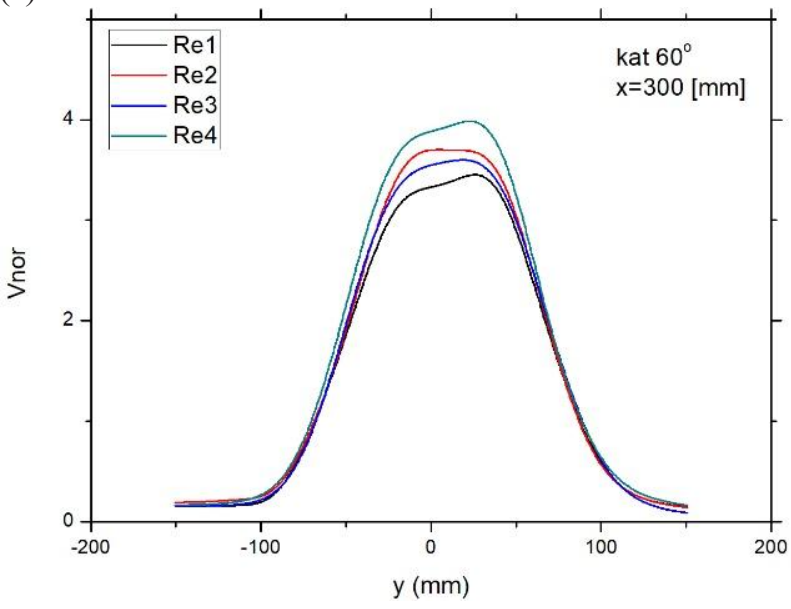

(f) $\mathrm{x}=400 \mathrm{~mm}$

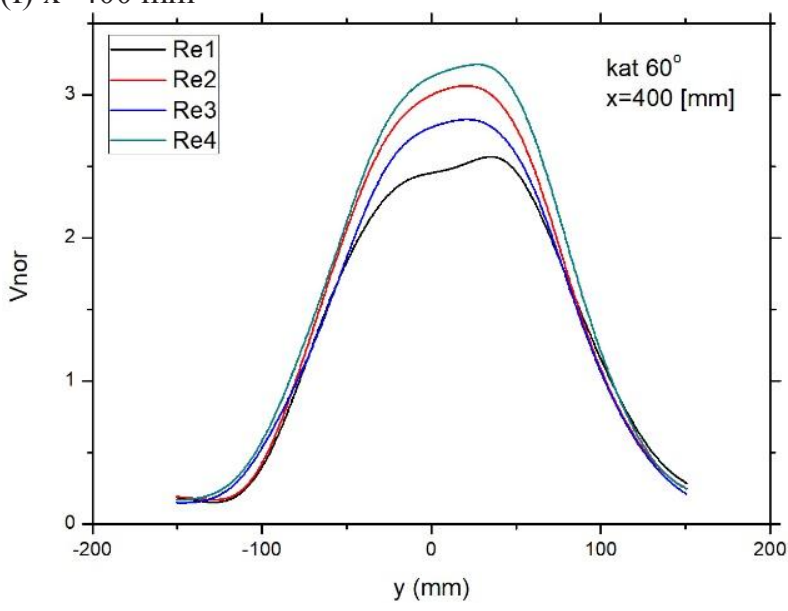

Fig. 8. Normalised velocity profiles of air velocity flowing out of the diffuser for diffuser vanes at an angle $60^{\circ}$ and at distances (a) $x=70 \mathrm{~mm}$; (b) $x=100 \mathrm{~mm}$; (c) $x=150 \mathrm{~mm}$; (d) $x=200 \mathrm{~mm}$; (e) $x=300 \mathrm{~mm}$; (f) $x=400 \mathrm{~mm}$ from the outlet of the diffuser.

The degree of conformity of distributions of a given size $c$, for subsequent ( $i$ and $i+1)$ numbers Re can be expressed in quantity by the mean relative difference:

$$
\alpha_{w}=\frac{\frac{1}{n} \sum_{i=1}^{n}\left|C_{R \theta_{i+1}}-C_{R \theta_{i}}\right|}{\frac{1}{n} \sum_{i=1}^{n}\left|C_{R \epsilon_{i+1}}\right|}=100 \%
$$

where:

$C_{R e_{1}}-$ value of the value $\mathrm{c}$ (in this cave velocity magnitude) measured for the $\mathrm{i}$-th number $\mathrm{Re}_{\mathrm{i}}: \mathrm{Re}_{1}=47,000.00, \mathrm{Re}_{2}=$ $85,000.00, \mathrm{Re}_{3}=143,000.00, \mathrm{Re}_{4}=183,000.00$.

Table 1 shows the values of $\alpha_{w}$ calculated for a diffuser with an angle of $60^{\circ}$. Measurements were made at different values of Reynolds numbers. The lowest value of $\alpha_{w}$ was $8.1 \%$ and the highest $43 \%$. For the highest Reynolds number, the value of the indicator record the average value about $14.6 \%$. For higher values of the number Re, the coefficient $\alpha_{w}$ reaches the lowest values for profiles plotted at distances of $200 \mathrm{~mm}, 300 \mathrm{~mm}$ and $400 \mathrm{~mm}$ from the diffuser outlet.
Table 1. The relative difference of velocity vector distributions for a diffuser with vanes at an angle of $60^{\circ}$.

\begin{tabular}{|c|c|c|c|}
\hline & \multicolumn{3}{|c|}{$\alpha_{w}$} \\
\hline Section & $\operatorname{Re} 1 \rightarrow \operatorname{Re} 2$ & $\operatorname{Re} \rightarrow \operatorname{Re} 3$ & $\operatorname{Re} 3 \rightarrow \operatorname{Re} 4$ \\
\hline $\mathrm{x}=70 \mathrm{~mm}$ & $21.3 \%$ & $43.0 \%$ & $20.6 \%$ \\
\hline $\mathrm{x}=100 \mathrm{~mm}$ & $9.4 \%$ & $35.4 \%$ & $17.2 \%$ \\
\hline $\mathrm{x}=150 \mathrm{~mm}$ & $16.3 \%$ & $23.2 \%$ & $15.0 \%$ \\
\hline $\mathrm{x}=200 \mathrm{~mm}$ & $17.0 \%$ & $20.3 \%$ & $11.8 \%$ \\
\hline $\mathrm{x}=300 \mathrm{~mm}$ & $8.1 \%$ & $11.1 \%$ & $12.0 \%$ \\
\hline $\mathrm{x}=400 \mathrm{~mm}$ & $10.6 \%$ & $9.2 \%$ & $11.1 \%$ \\
\hline Average value & $13.8 \%$ & $23.7 \%$ & $14.6 \%$ \\
\hline
\end{tabular}

\section{Numerical model}

The numerical analysis of airflow by swirl diffuser was conducted using Computational Fluid Dynamics technique (CFD) and specialized software Star-CCM+ [11]. The 3D geometrical model, presented in Figure 9, consist of swirler, diffuser and surrounding area, created to calculate velocity profiles of air flowing out of the diffuser.

The boundary conditions in the form of the inlet, outlet and wall were implemented as is presented in Figure 9. The geometrical model was divided into 619382 polyhedral cells, with four boundary layers close to the concave wall.

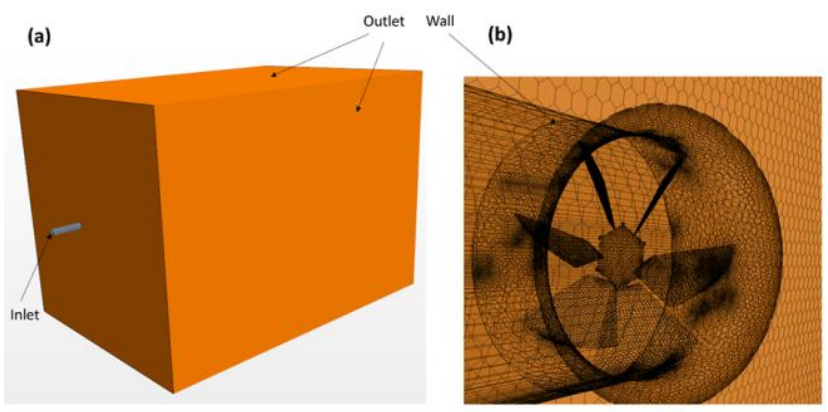

Fig. 9. Geometrical model of analysed region with boundary conditions used in the simulation (a) and numerical mesh close to the diffuser region (b).

The simulations were conducted in steady-state, using air as non-compressible fluid at ambient conditions (Table 2 ). The turbulent character of flow was simulated using realizable two-layer k- $\varepsilon$ turbulence model. This model is a two-equation model that solves transport equations for the turbulent kinetic energy $\mathrm{k}$ and the turbulent dissipation rate $\varepsilon$ in order to determine the turbulent eddy viscosity $\mu$ t. 
General forms of equations used in $\mathrm{k}-\varepsilon$ turbulence model are as follows:

$$
\mu_{t}=\rho C_{\mu} f_{\mu} k T
$$

$\frac{\partial}{\partial t}(\rho k)+\nabla \cdot(\rho k \overline{\mathbf{v}})=\nabla \cdot\left[\left(\mu+\frac{\mu_{\mathrm{k}}}{\sigma_{k}}\right) \nabla k\right]+P_{k}-\rho(\varepsilon-$ $\left.\epsilon_{0}\right)+S_{k}$

$$
\begin{aligned}
& \frac{\partial}{\partial t}(\rho \varepsilon)+\nabla \cdot(\rho \varepsilon \overline{\mathbf{v}})=\nabla \cdot\left[\left(\mu+\frac{\mu_{E}}{\sigma_{E}}\right) \nabla \varepsilon\right]+\frac{1}{T_{I E}} C_{\varepsilon 1} P_{\varepsilon}- \\
& C_{\varepsilon 2} f_{2} \rho\left(\frac{\varepsilon}{T_{E}}-\frac{E_{0}}{T_{0}}\right)+S_{\varepsilon}
\end{aligned}
$$

where: $\rho$ - density $\left(\mathrm{kg} / \mathrm{m}^{3}\right) ; T$ - turbulent time scale (s); $\overline{\boldsymbol{v}}-$ mean velocity $(\mathrm{m} / \mathrm{s}), \boldsymbol{\mu}-$ dynamic viscosity $(\mathrm{Pa} \cdot \mathrm{s})$, $C_{\mu}, \sigma_{k}, \sigma_{\varepsilon}, C_{\varepsilon 1}, C_{\varepsilon 2}-$ model coefficients, $f_{\mu}, f_{2}-$ damping functions, $P_{k}, P_{\xi}-$ production terms, $S_{k}, S_{E}-$ user-specified source terms.

In the two-layer approach, in layer close to the wall turbulent dissipation rate and turbulent viscosity are specified using functions of wall, giving good accuracy in simulation with low and high Reynolds number.

Table 2. Boundary conditions and air properties used in simulation.

\begin{tabular}{|c|c|c|c|c|}
\hline No & $\begin{array}{c}\text { Density, } \\
\mathrm{kg} / \mathrm{m}^{3}\end{array}$ & $\begin{array}{c}\text { Dynamic } \\
\text { viscosity, } \\
\mathrm{Pa} \cdot \mathrm{s}\end{array}$ & $\begin{array}{c}\text { Mass flow } \\
\text { rate, } \mathrm{kg} / \mathrm{s}\end{array}$ & $\begin{array}{c}\text { Volumetric } \\
\text { flow rate, } \\
\mathrm{m}^{3} / \mathrm{h}\end{array}$ \\
\hline 1 & 1.18415 & $1.85508 \mathrm{e}-05$ & 0.0327 & 100 \\
\hline
\end{tabular}

The conducted simulation allows to obtain results in form of velocity distribution in a vertical plane and compare this filed with measuring results using PIV. In Figure 10 the distribution of velocity magnitude is presented, and in Figure 11 the velocity vectors are possible to compare with measured values of velocity vectors.

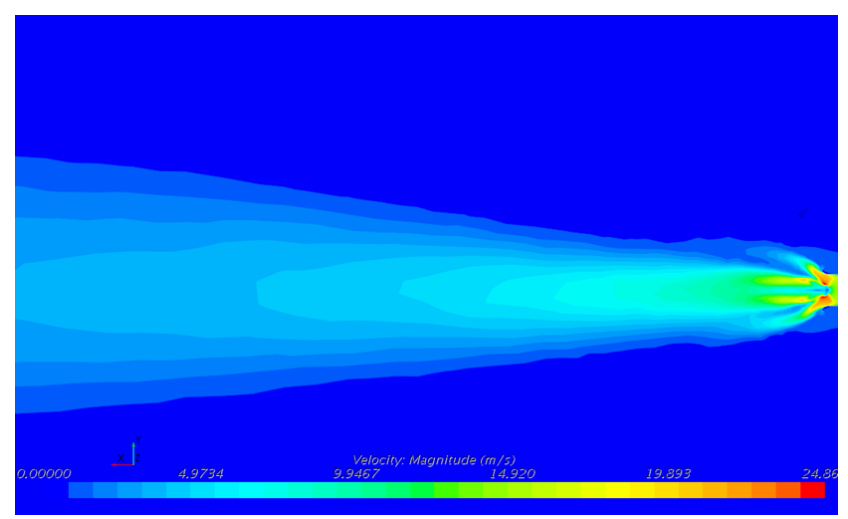

Fig. 10. The results of calculated velocity distribution. For the case: $60^{\circ}$ diffuser and flowrate $100 \mathrm{~m}^{3} / \mathrm{h}$.

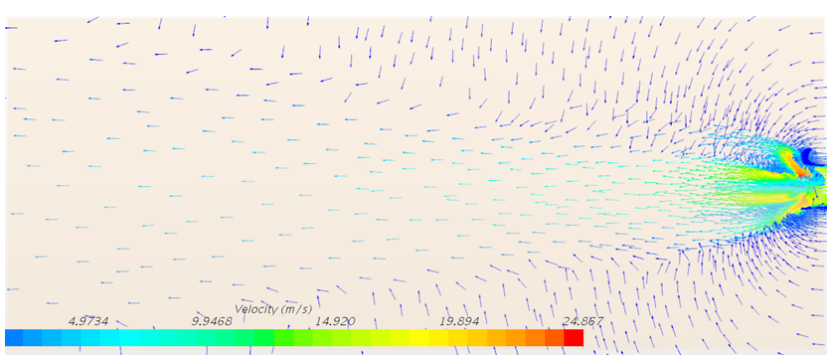

Fig. 11. The results of calculated velocity vectors in vertical plane $x-y$ (Fig. 5). For the case: $60^{\circ}$ diffuser and flow rate 100 $\mathrm{m}^{3} / \mathrm{h}$.

\section{Conclusions}

Application of Particle Image Velocimetry measurements showed the advantages of this method over "standard" methods in areas related to flow field determination in ventilated area. For example, the evaluating the range of the air stream using thermoanemometric sensors required large number of analysis. Simultaneous measurement in a large flow points. The ability to evaluate velocity vectors simultaneously at any point in this flow determines the value and suitability of the measurement method used.

The results of field velocity measurements were analysed for various value of the Reynolds number. The normalised velocity profiles at different cross-sections $x-y$ of the stream were presented and compared. The relative difference of distributions of the velocity vector values was calculated. This ratio decreases with the increase in the Reynolds number. Its lowest values were obtained for the flows with the Reynolds number 143,000.00 and above. With the increase in the Reynolds number, the degree of conformity of normalized distributions of velocity fields increases. The presented laboratory model of a swirl diffuser, for turbulent flow at Reynolds number higher than $143,000.00$ has the ability to self- similarity model.

The present work was partially supported by the Polish Ministry of Science (Grant AGH No. 11.11.100.920).

\section{References}

1. A. Melikov, C. Radim, M. Milan, Personalized ventilation: evaluation of different air terminal devices, Energy Buildings, 34, pp. 829-36 (2002)

2. M. Jaszczur, M. Borowski, M. Karch, M. Branny, The study of the velocity field of the air flowing the swirl diffusers using PIV method, EPJ Web of Conferences, 143 (2017)

3. M.A. Aziz et al, Experimental and numerical study of influence of air ceiling diffusers on room air, Energy Buildings, 55 (2012)

4. E. Tavakoli, R. Hosseini, Large eddy simulation of turbulent flow and mass transfer in far-field of swirl diffusers Energy Buildings, 59 (2013)

5. M. Jaszczur et al, Experimental analysis of the velocity field of the air flowing through the swirl diffusers, Journal of Physics: Conference Series 745, 032049 (2016) 
6. M. Borowski, M. Jaszczur, D. Satoła, M. Karch, Air flow characteristics of a room with air vortex diffuser MATEC Web of Conferences, ISSN 2261236X., vol. 240 art. no. 02002, s. 1-5 (2018)

7. Z. Liu, Q. Li, T. Ishihara, Numerical investigation of a swirl diffuser with a novel design using large eddy simulations, Building and Environment, 135 (2018)

8. C.S. From, E. Sauret, S.W. Armfield, S.C. Saha, Y.T. Gu, Turbulent dense gas flow characteristics in swirling conical diffuser, Comput. Fluids 149 (2017)

9. Y.H. Yau, K.S. Poh, A. Badarudin, A numerical airflow pattern study of a floor swirl diffuser for UFAD system, Energy Build, 158 (2018)

10. A. Li, C. Yang, T. Ren, X. Bao, E. Qin, R. Gao, PIV experiment and evaluation of air flow performance of swirl diffuser mounted on the floor, Building and Environment, 135 (2018)

11. Ansys Inc, Ansys Fluent in Ansys Workbench User's Guide. U.S. (2012) 